\title{
Analysis of amplitude modulation atomic force microscopy in aqueous salt solutions
}

\author{
Pinar Karayaylalı ${ }^{\mathrm{a}}$, Mehmet Z. Baykara ${ }^{\mathrm{a}, \mathrm{b}, *}$ \\ a Department of Mechanical Engineering, Bilkent University, Ankara 06800, Turkey \\ ${ }^{\mathrm{b}}$ UNAM-Institute of Materials Science and Nanotechnology, Bilkent University, Ankara 06800, Turkey
}

\section{A R T I C L E I N F O}

\section{Article history:}

Received 13 November 2013

Received in revised form 15 January 2014

Accepted 6 February 2014

Available online 19 February 2014

Keywords:

Atomic force microscopy

Imaging of biomaterials

Electrostatic double layer forces

\begin{abstract}
A B S T R A C T
We present a numerical analysis of amplitude modulation atomic force microscopy in aqueous salt solutions, by considering the interaction of the microscope tip with a model sample surface consisting of a hard substrate and soft biological material through Hertz and electrostatic double layer forces. Despite the significant improvements reported in the literature concerning contact-mode atomic force microscopy measurements of biological material due to electrostatic interactions in aqueous solutions, our results reveal that only modest gains of $\sim 15 \%$ in imaging contrast at high amplitude setpoints are expected under typical experimental conditions for amplitude modulation atomic force microscopy, together with relatively unaffected sample indentation and maximum tip-sample interaction values.
\end{abstract}

(C) 2014 Elsevier B.V. All rights reserved.

\section{Introduction}

Since its invention more than two decades ago, atomic force microscopy (AFM) has become the most widely utilized member of the scanning probe microscopy family in research and industrial laboratories around the world $[1,2]$. A key factor in the widespread use of AFM is its ability to image material surfaces with (sub)-nm resolution in a large number of environmental conditions, ranging from ultrahigh vacuum (UHV) to ambient and liquids. While imaging in UHV using certain operational modes of AFM has allowed atomic-resolution imaging of atomically flat and clean surfaces [3], the main motivation behind operating in liquids has been the goal of high-resolution imaging of biological material such as cell membranes, DNA, and various fibrous and globular proteins in their natural states, without structural deformations caused by vacuum conditions needed for transmission electron microscopy (TEM), the traditional method of choice for high-resolution imaging of biomaterials [4-6].

AFM has been initially used in the contact-mode in liquids to image biomaterials such as purple membrane and DNA [7,8]. In this common mode of AFM, a micro-machined cantilever with a sharp tip [9] is brought into soft contact with the sample surface under investigation (with contact forces on the order of a few $\mathrm{nN}$ ) and scanned laterally with pm precision while vertical deflections

\footnotetext{
* Corresponding author at: Department of Mechanical Engineering, Bilkent University, Ankara 06800, Turkey. Tel.: +90 3122903428.

E-mail address: mehmet.baykara@bilkent.edu.tr (M.Z. Baykara).
}

of the cantilever caused by topographical features of the sample surface are detected, mostly by using the laser beam deflection (LBD) method [10]. Thus, high resolution maps of biological materials may be obtained in liquids such as pure water or phosphate buffer solution (PBS). One major drawback of contact-mode imaging of biological matter is the occurrence of lateral forces between the probe tip and the sample during imaging, frequently damaging and displacing the soft biological matter under investigation [4]. To circumvent this problem, Müller et al. have successfully demonstrated the use of repulsive electrostatic interaction forces occurring between the probe tip and the sample surface in aqueous salt solutions due to accumulated surface charges [11]. Thus, attractive interaction forces acting locally between the tip apex and sample at close separations are electrostatically balanced and sample deformation is significantly reduced with a noticeable increase in resolution.

An alternative method to reduce the influence of lateral forces on biological material during imaging in liquids is to employ dynamic imaging modes of AFM [12,13]. In dynamic AFM, the cantilever with the probe tip is oscillated at or near its resonance frequency using various actuation methods [14-16] and changes in its oscillation characteristics (such as amplitude, phase or frequency) due to tip-sample interactions are recorded. While frequency modulation atomic force microscopy (FM-AFM, where the oscillation amplitude is kept constant during imaging and changes in oscillation frequency are detected) has recently been employed to perform molecular resolution imaging of biomaterials in liquids thanks to several advances in instrumentation [17-21], amplitude modulation atomic force microscopy (AM-AFM, where 


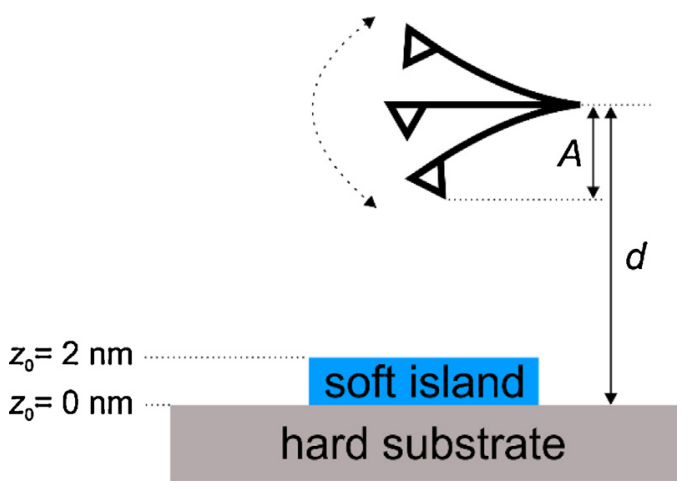

Fig. 1. Schematic describing the model used in the numerical simulations. The cantilever is oscillating with an amplitude of $A$ while its base is located a distance $d$ above the hard substrate. The height of the soft island is taken to be $2 \mathrm{~nm}$.

the excitation frequency is kept constant during imaging and changes in oscillation amplitude are detected) is usually preferred due to its relative technical simplicity [22]. Accordingly, AM-AFM (often referred to as tapping-mode AFM) has been used to image a number of biomaterials in liquids in the past $[23,24]$. It should be indicated that the main experimental challenge associated with AM-AFM imaging in liquids is the significantly reduced $Q$-factor of the cantilever, leading to low signal-to-noise ratios [25]. As such, attempts to improve the effective $Q$-factor such as the method of $Q$-Control have been employed in the past, leading to improved imaging contrast, as well as reduced sample deformation and interaction forces $[26,27]$.

Being inspired by the advances in AFM measurement of biomaterials in liquids summarized above, we have investigated in this contribution the effect of operating in aqueous salt solutions on AM-AFM imaging of a model biological sample using numerical simulations. Contrary to contact-mode operation, our results indicate very modest gains in imaging contrast due to electrostatic interactions at high amplitude setpoints, accompanied by relatively unaffected sample indentation and maximum tip-sample interaction values.

\section{Theoretical considerations and modeling}

AM-AFM operation in liquid conditions has been numerically and theoretically analyzed in a number of studies in the past [27-30]. Most commonly, the equation of motion for the oscillating cantilever is considered to be in the following form:

$m \ddot{z}(t)+\frac{2 \pi f_{0} m}{Q} \dot{z}(t)+k(z(t)-d)=k A_{\text {ext }} \cos \left(2 \pi f_{\text {ext }} t\right)+F_{\text {total }}(z(t))$

where $m$ is the effective mass of the cantilever, $z(t)$ the position of the oscillating tip of the cantilever relative to the sample surface at time $t, f_{0}$ the resonance frequency of the cantilever $\left(f_{0}=1 / 2 \pi \sqrt{k / m}\right), Q$ the quality factor, $k$ the spring constant and $d$ the distance of the cantilever base to the sample surface. The cantilever is oscillated mechanically (e.g., using a piezoelectric element) with a constant driving amplitude of $A_{\text {ext }}$ and a constant driving frequency of $f_{\text {ext. }} F_{\text {total }}(z(t))$ is the total interaction force acting between the tip and the sample surface at position $z(t)$.

As a model sample system appropriate for simulating AM-AFM experiments in liquids on biological material, we have considered a soft $\left(E_{\mathrm{s}, \mathrm{soft}}=1 \mathrm{GPa}\right)$ island of $2 \mathrm{~nm}$ height on top of a hard substrate $\left(E_{\mathrm{s}, \text { hard }}=130 \mathrm{GPa}\right)$, in accordance with previous studies [27] (see Fig. 1). The height of the soft island roughly coincides with that of DNA, while the elastic modulus of the substrate follows that of silicon $(\mathrm{Si})$, based on the fact that DNA adsorbed on Si or mica substrates are frequently used as test samples for liquid AM-AFM experiments [4].

When performing AFM measurements in deionized liquids, attractive interactions including van der Waals' forces are greatly reduced due to screening $[27,31,32]$ and the main interaction force is due to the elastic contact between the probe tip and the sample surface which is appropriately described by Hertzian contact theory [33] as follows:

$F_{H}(z)=\frac{4}{3} E^{\prime} \sqrt{R}\left(z_{0}-z\right)^{3 / 2}$

where $E^{\prime}$ is a parameter derived from Young's modulus and Poisson's ratio values for the tip and sample $\left(E_{\mathrm{t}}, E_{\mathrm{s}}, \mu_{\mathrm{t}}, \mu_{\mathrm{s}}\right)$ such that $E^{\prime}=\left[\left(1-\mu_{\mathrm{s}}^{2}\right) / E_{\mathrm{s}}+\left(1-\mu_{\mathrm{t}}^{2}\right) / E_{\mathrm{t}}\right]^{-1}, R$ the radius of the AFM tip modeled as a sphere and $z_{0}$ a constant value describing the height of the sample surface (for our model sample system, $z_{0}=2 \mathrm{~nm}$ for the soft island and $z_{0}=0$ for the hard substrate). Naturally, repulsive contact forces described by $F_{\mathrm{H}}$ affect cantilever motion only when contact between tip and sample occurs (i.e., $\left.\left(z_{0}-z\right)>0\right)$. For noncontact conditions $\left(\left(z_{0}-z\right) \leq 0\right), F_{H}$ becomes zero. It should be noted here that the accuracy of Hertzian contact forces calculated in our simulations are limited by assumptions involving linear elasticity, isotropy and homogeneity, among others. While linearly elastic conditions may not always be satisfied during actual AM-AFM measurements performed on biological material, Hertzian contact theory has been used in the literature to successfully estimate contact forces to a first approximation in such cases [22,27]. Thus, it has been employed in the present discussion as well for reasons of comparability. Moreover, hydrodynamic reaction forces which are comparably small for typical cantilever tip dimensions as well as solvation forces have been neglected in our analysis in accordance with previous AM-AFM simulation work in liquids [22,27].

When performing AM-AFM measurements in aqueous salt solutions, both the AFM tip and the sample develop a net surface charge, based on various mechanisms such as the dissociation of certain surface groups and adsorption of ions onto the material surface [34]. Due to the electrostatic interaction between the charged surfaces and the ions in the salt solution, a concentration gradient called the electrical double layer (EDL) exists near the immersed surfaces. An electrical double layer force $\left(F_{\mathrm{EDL}}\right)$ based on mutually attractive or repulsive electrostatic interactions is thus observed between sample and tip when the distance between them is on the order of a few tens of nanometers. While the Poisson-Boltzmann theoretical framework provides an accurate description of the potential that develops between such surfaces and the associated interaction forces [35], it involves the numerical solution of a second order nonlinear differential equation, complicating its usefulness. Alternatively, an approximate form of the EDL force that develops between a planar and a spherical surface (such as the sample and the tip surfaces in an AFM experiment) may be used as [36]

$F_{\mathrm{EDL}}(z)=\left(\frac{4 \pi R \sigma_{\mathrm{s}} \sigma_{\mathrm{t}}}{\varepsilon_{0} \varepsilon}\right) \delta \exp \left(\frac{z_{0}-z}{\delta}\right)$

for $\left(z_{0}-z\right) \leq 0$, where $\sigma_{\mathrm{s}}$ and $\sigma_{\mathrm{t}}$ are surface charge densities of sample and tip, respectively, $\varepsilon_{0}$ the permittivity of vacuum, $\varepsilon$ the dielectric constant of the liquid and $\delta$ the Debye length, described by:

$\delta=\sqrt{\left(\frac{\varepsilon_{0} \varepsilon k_{\mathrm{B}} T}{e^{2} \sum_{i} c_{i} Z_{i}}\right)}$

where $k_{\mathrm{B}}$ is the Boltzmann constant, $T$ the temperature, $e$ the electronic charge, $c_{i}$ the concentration of the $i$ th type of ion in the salt solution and $Z_{i}$ the valence value for the same ion type. While it should be indicated that the approximate form of the EDL force 
provided by Eq. (3) is of limited accuracy once the distance between the surfaces is below the Debye length, it has been successfully implemented in a number of AFM studies in the past, and has thus been adopted for the present discussion as well [36-38]. Finally, combining the Hertzian contact force and the EDL force, the total tip-sample interaction force is obtained as

$F_{\text {total }}=F_{\mathrm{EDL}}(z)=\left(\frac{4 \pi R \sigma_{\mathrm{s}} \sigma_{\mathrm{t}}}{\varepsilon_{0} \varepsilon}\right) \delta \exp \left(\frac{z_{0}-z}{\delta}\right)$ when $\quad z \geq z_{0}$

$F_{\text {total }}=F_{H}(z)+F_{\mathrm{EDL}}(0)=\frac{4}{3} E^{\prime} \sqrt{R}\left(z_{0}-z\right)^{3 / 2}+\left(\frac{4 \pi R \sigma_{\mathrm{s}} \sigma_{\mathrm{t}}}{\varepsilon_{0} \varepsilon}\right) \delta$

when $z<z_{0}$

Experimentally appropriate parameters used in the simulations for the above described model sample surface and a typical Si cantilever are as follows: $E_{\mathrm{t}}=130 \mathrm{GPa}, \mu_{\mathrm{t}}=\mu_{\mathrm{s}, \mathrm{soft}}=\mu_{\mathrm{s} \text {, hard }}=0.3$, $\sigma_{\mathrm{t}}=\sigma_{\mathrm{s}, \mathrm{hard}}=-0.012 \mathrm{C} / \mathrm{m}^{2}, \sigma_{\mathrm{s}, \mathrm{soft}}=-0.04 \mathrm{C} / \mathrm{m}^{2}, T=293 \mathrm{~K}, \varepsilon=80.2$, $R=10 \mathrm{~nm}, f_{0}=20 \mathrm{kHz}, Q=5, k=1 \mathrm{~N} / \mathrm{m}, f_{\text {ext }}=f_{0}=20 \mathrm{kHz}$. $A_{\text {ext }}$ is chosen such that the cantilever undergoes a free oscillation amplitude of $A_{0}=10 \mathrm{~nm}$ far from the sample surface when tip-sample interactions are negligible, similar to earlier simulation work [27]. Please note $f_{0}=20 \mathrm{kHz}$ corresponds to the wet resonance frequency of the cantilever in the liquid medium [30] and does not imply unusually large dimensions. It should be noted that while it has been recently demonstrated that atomic-resolution imaging of mineral surfaces such as mica is made possible by a significant reduction of oscillation amplitude in liquids [39], and the use of small, high-frequency cantilevers in conjunction with high-speed AFM leads to impressive results [40,41], typical experimental parameters for imaging biomaterials using AM-AFM remain similar to the values employed in our simulations. Tip and surface charge density values for our model system - which generally display a rather weak dependence on salt concentration down to $1 \mathrm{mM}$ [35] and have thus been taken to be constant in this study - have been determined based on experimental work in the literature $[35,36]$ and result in a net repulsive EDL force. All results presented in Section 3 have been obtained by numerically solving Eq. (1) for the variable $z(t)$ by applying a fourth order Runge-Kutta method for set values of $d$, representing fixed distances between the cantilever base and sample surface.

\section{Results and discussion}

In typical AM-AFM operation, the cantilever is driven with a fixed driving amplitude $\left(A_{\text {ext }}\right)$ and a fixed driving frequency $\left(f_{\text {ext }}\right)$, while shifts in the oscillation amplitude $(A)$ with decreasing tip-sample distance due to increasing force interactions are detected. Imaging is usually performed at a fixed amplitude setpoint (usually $10 \%$ to $20 \%$ lower than the free oscillation amplitude $A_{0}$ ) by the utilization of a feedback loop. As such, the imaging contrast between different regions of a sample surface are determined by the vertical displacement of the cantilever base required to keep the amplitude setpoint constant during imaging. Therefore,
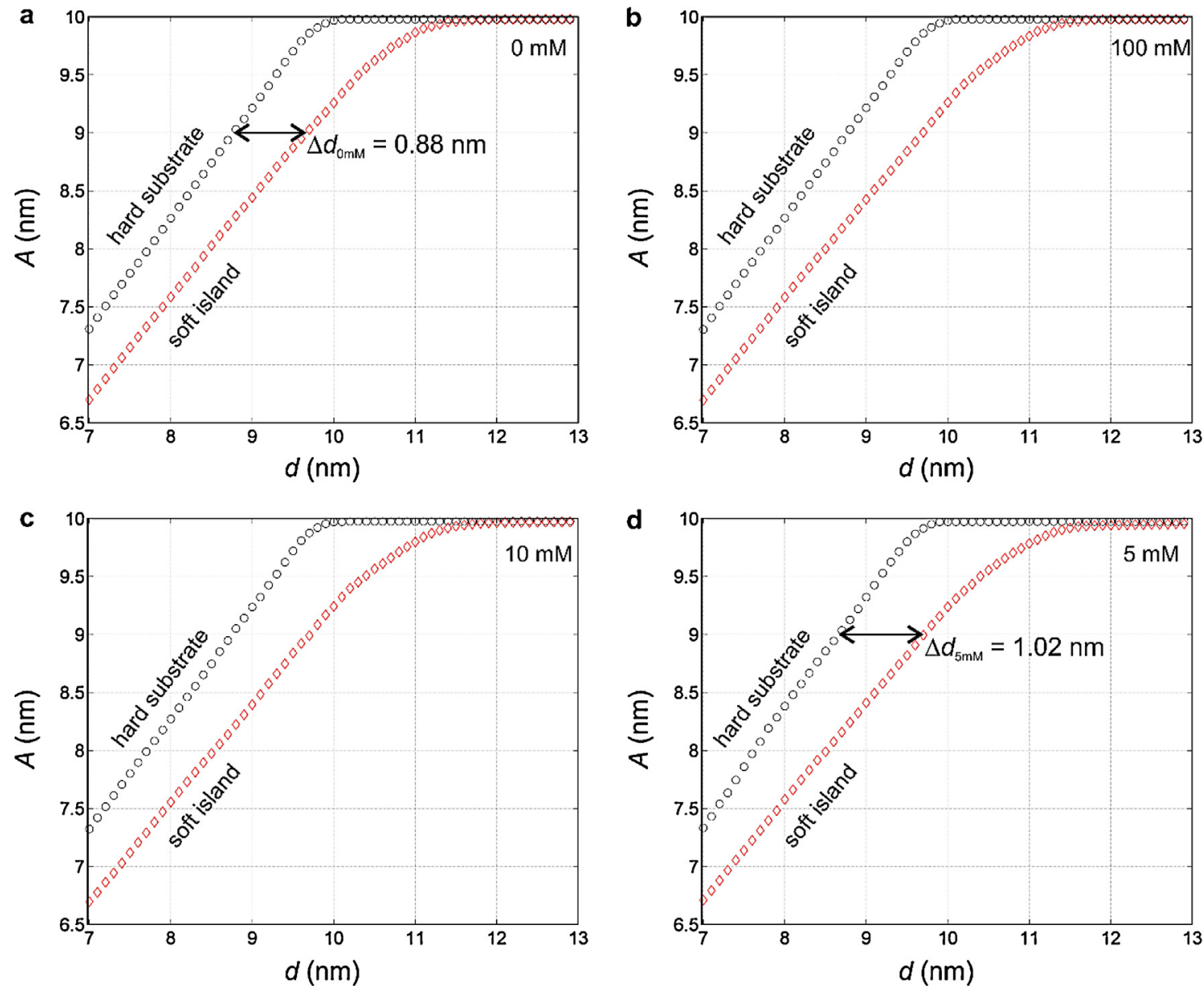

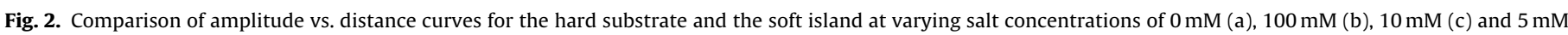

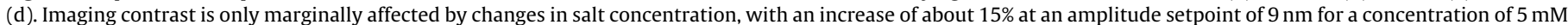

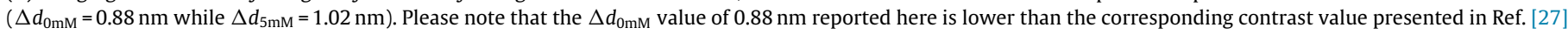
due to a difference in the Young's Moduli of substrates employed in the respective simulations. 
it would be appropriate to compare amplitude vs. distance ( $A$ vs. $d$ ) curves for the hard substrate and the soft island employed in our model sample surface for varying salt concentrations to investigate the effect of operating in aqueous salt solutions on imaging performance of AM-AFM in liquids. Accordingly, numerically obtained $A$ vs. $d$ curves for monovalent salt concentrations of $0 \mathrm{mM}, 5 \mathrm{mM}$, $10 \mathrm{mM}$, and $100 \mathrm{mM}$ are provided in Fig. 2 for a distance $(d)$ regime of $7-13 \mathrm{~nm}$. The reason for the consideration of monovalent (e.g., $\mathrm{NaCl}, \mathrm{KCl}$ ) instead of divalent (e.g., $\mathrm{MgCl}_{2}, \mathrm{CaCl}_{2}$ ) salt species in the present calculations is that the EDL forces caused by equal concentrations of monovalent salts are found to be significantly higher than divalent salts, based on higher Debye lengths [35]. As such, monovalent salt species are more useful for assessing the effects of electrostatic interactions on AM-AFM operation in liquids. Let us note here that a salt concentration of $0 \mathrm{mM}$ corresponds to the completely deionized case where the EDL contribution to the total force interaction is zero.

Comparing the plots in Fig. 2, two main conclusions are made:

(1) As expected from experimental work in the literature [11,35], the effect of salt solutions on $A$ vs. $d$ curves is strongest at low salt concentrations such as $5 \mathrm{mM}$ due to increased Debye lengths. Consequently, the effect of salt solutions on $A$ vs. $d$ curves are negligible at high concentrations such as $100 \mathrm{mM}$.

(2) Even for low salt concentrations of, e.g., $5 \mathrm{mM}$, the effect of EDL forces on $A$ vs. $d$ behavior is small, resulting in an increase of only about $15 \%$ in height contrast $(\Delta d)$ between the hard substrate and the soft island at a relatively high setpoint amplitude of $A=9 \mathrm{~nm}$. As expected, the modest increase in contrast due to the earlier onset of EDL forces for the soft island (both due to the fact that the soft island is closer to the tip than the hard substrate by $2 \mathrm{~nm}$ and the fact that the surface charge density is higher on the soft island) diminishes with increasing salt concentration. Compared to the increase in height contrast of more than $60 \%$ provided by the method of $Q$-Control on a very similar sample system [27], it is clear that operation in aqueous salt solutions does not lead to a significant improvement in imaging contrast for AM-AFM, despite the fact that differences in surface charge density have resulted in detectable differences in the phase shift signal in an earlier study in the literature [42].

The reason for the marginal effect of EDL interactions on AMAFM imaging becomes clear when the maximum contributions of the EDL $\left(F_{\mathrm{EDL}}\right)$ and Hertz $\left(F_{H}\right)$ interactions to the total tip-sample interaction $\left(F_{\text {total }}\right)$ are compared for the soft island in our model sample system. Even for a relatively low salt concentration of $5 \mathrm{mM}$, the maximum value for $F_{\mathrm{EDL}}(\sim 0.4 \mathrm{nN})$ is more than an order of magnitude lower than that observed for the contact force $F_{H}(\sim 10 \mathrm{nN})$ in the investigated distance regime. As such, the tip-sample interaction is mainly dominated by contact forces during AM-AFM operation in aqueous salt solutions, limiting the effect of electrostatic interactions on imaging. It should be noted that the calculated maximum values for the EDL interaction are in good quantitative agreement with experimental results reported in the literature for monovalent salts (taking into account the differences in tip radius and sample surface charge density) [35] despite the relatively basic nature of our model sample system and calculations.

Another aspect that needs to be considered when evaluating AM-AFM measurements in liquids on biological material is the issue of sample deformation. Since typically the biological material to be imaged is mechanically much weaker than the substrate it is adsorbed on, low interaction forces and indentation values are desirable. The results of the present numerical analysis indicate that maximum tip-sample interaction forces only marginally increase (again due to the significantly lower magnitude of EDL forces when compared to contact forces) while sample indentation
Table 1

Comparison of maximum interaction force and sample indentation values for the soft island in our model sample system at varying salt concentrations $(d=7 \mathrm{~nm})$ It is readily observed that sample indentation values are essentially unaffected by changes in salt concentration, while maximum interaction forces only marginally increase with decreasing salt concentration when compared to the deionized liquid.

\begin{tabular}{lll}
\hline Salt concentration $(\mathrm{mM})$ & $\begin{array}{l}\text { Maximum interaction } \\
\text { force }(\mathrm{nN})\end{array}$ & $\begin{array}{l}\text { Sample indentation } \\
(\mathrm{nm})\end{array}$ \\
\hline 0 & 10.2 & 1.7 \\
100 & 10.2 & 1.7 \\
10 & 10.4 & 1.7 \\
5 & 10.5 & 1.7 \\
\hline
\end{tabular}

values remain relatively unchanged with decreasing salt concentration (see Table 1) when compared to imaging in deionized liquids.

\section{Conclusions}

In summary, we have performed a model numerical analysis of amplitude modulation atomic force microscopy on soft biological materials adsorbed on hard substrates in aqueous salt solutions. Despite the significant advantages provided by repulsive electrostatic interactions in contact-mode imaging of similar samples [11], our results indicate that only modest gains in imaging contrast at high amplitude setpoints are expected for AM-AFM under typical experimental conditions represented by our simulations, while sample indentation and maximum tip-sample interaction values remain relatively unaffected.

\section{References}

[1] G. Binnig, C.F. Quate, C. Gerber, Phys. Rev. Lett. 56 (1986) 930.

[2] P.J. Eaton, P. West, Atomic Force Microscopy, Oxford University Press, Oxford, 2010.

[3] S. Morita, R. Wiesendanger, E. Meyer, Noncontact Atomic Force Microscopy Springer, Berlin, 2002

[4] V.J. Morris, A.R. Kirby, A.P. Gunning, Atomic Force Microscopy for Biologists, Imperial College Press, London, 2010

[5] A.M. Baro, R.G. Reifenberger, Atomic Force Microscopy in Liquid: Biological Applications, Wiley-VCH, Weinheim, 2012

[6] P. Parot, Y.F. Dufrene, P. Hinterdorfer, C. Le Grimellee, D. Navajas, J.L. Pellequer, S. Scheuring, J. Mol. Recognit. 20 (2007) 418.

[7] D.J. Muller, F.A. Schabert, G. Buldt, A. Engel, Biophys. J. 68 (1995) 1681

[8] H.G. Hansma, J. Vesenka, C. Siegerist, G. Kelderman, H. Morrett, R.L. Sinsheimer, V. Elings, C. Bustamante, P.K. Hansma, Science 256 (1992) 1180.

[9] T.R. Albrecht, S. Akamine, T.E. Carver, C.F. Quate, J. Vac. Sci. Technol., A 8 (1990) 3386.

[10] G. Meyer, N.M. Amer, Appl. Phys. Lett. 53 (1988) 1045.

[11] D.J. Müller, D. Fotiadis, S. Scheuring, S.A. Muller, A. Engel, Biophys. J. 76 (1999 1101.

[12] R. Garcia, R. Perez, Surf. Sci. Rep. 47 (2002) 197.

[13] P.K. Hansma, et al., Appl. Phys. Lett. 64 (1994) 1738.

[14] M. Dreier, D. Anselmetti, T. Richmond, U. Dammer, H.J. Guntherodt, J. Appl Phys. 76 (1994) 5095.

[15] W.H. Han, S.M. Lindsay, T.W. Jing, Appl. Phys. Lett. 69 (1996) 4111

[16] D. Ramos, J. Tamayo, J. Mertens, M. Calleja, J. Appl. Phys. 99 (2006) 124904.

[17] H. Yamada, K. Kobayashi, T. Fukuma, Y. Hirata, T. Kajita, K. Matsushige, Appl. Phys. Express 2 (2009) 095007

[18] H. Asakawa, T. Fukuma, Nanotechnology 20 (2009) 264008

[19] H. Asakawa, T. Fukuma, Rev. Sci. Instrum. 80 (2009) 103703.

[20] T. Fukuma, Rev. Sci. Instrum. 80 (2009) 023707

[21] Y. Mitani, M. Kubo, K. Muramoto, T. Fukuma, Rev. Sci. Instrum. 80 (2009) 083705.

[22] R. Castro García, Amplitude Modulation Atomic Force Microscopy, Wiley-VCH, Weinheim, 2010.

[23] C. Moller, M. Allen, V. Elings, A. Engel, D.J. Muller, Biophys. J. 77 (1999) 1150.

[24] S. Kasas, et al., Biochemistry 36 (1997) 461.

[25] E.T. Herruzo, R. Garcia, Appl. Phys. Lett. 91 (2007) 143113

[26] D. Ebeling, H. Holscher, H. Fuchs, B. Anczykowski, U.D. Schwarz, Nanotechnology 17 (2006) S221.

[27] H. Holscher, U.D. Schwarz, Appl. Phys. Lett. 89 (2006) 073117.

[28] J. Legleiter, T. Kowalewski, Appl. Phys. Lett. 87 (2005) 163120

[29] S. de Beer, D. van den Ende, F. Mugele, Appl. Phys. Lett. 93 (2008) 253106.

[30] S. Basak, A. Raman, Appl. Phys. Lett. 91 (2007) 064107.

[31] J. Israelachvili, Intermolecular and Surface Forces, Academic Press, London, 1991.

[32] F. Ohnesorge, G. Binnig, Science 260 (1993) 1451 
[33] H. Hertz, J. Reine Angew. Math. 92 (1881) 156.

[34] H.J. Butt, Biophys. J. 60 (1991) 1438.

[35] D. Ebeling, D. van den Ende, F. Mugele, Nanotechnology 22 (2011) 305706.

[36] J. Sotres, A.M. Baro, Biophys. J. 98 (1995) (2010).

[37] J. Sotres, A. Lostao, C. Gomez-Moreno, A.M. Baro, Ultramicroscopy 107 (2007) 1207
[38] H.J. Butt, Biophys. J. 63 (1992) 578.

[39] D. Ebeling, S.D. Solares, Nanotechnology 24 (2013) 135702.

[40] N. Kodera, D. Yamamoto, R. Ishikawa, T. Ando, Nature 468 (2010) 72

[41] I. Casuso, et al., Nat. Nanotechnol. 7 (2012) 525

[42] D.M. Czajkowsky, M.J. Allen, V. Elings, Z.F. Shao, Ultramicroscopy 74 (1998) 1. 